\title{
RESET
}

Recherches en sciences sociales sur Internet

$10 \mid 2021$

Savoirs incertains

\section{Du tritium dans les gros titres}

Rumeurs anxiogènes et remise en cause des médias sur Twitter

Tritium in the headlines. Alarming rumors and media blaming on Twitter

\section{Antonin Segault}

\section{CpenEdition}

Journals

Édition électronique

URL : https://journals.openedition.org/reset/3185

DOI : $10.4000 /$ reset.3185

ISSN : 2264-6221

Éditeur

Association Recherches en sciences sociales sur Internet

Référence électronique

Antonin Segault, "Du tritium dans les gros titres », RESET [En ligne], 10 | 2021, mis en ligne le 13 mai 2021, consulté le 22 mai 2021. URL : http://journals.openedition.org/reset/3185 ; DOI : https://doi.org/ $10.4000 /$ reset.3185

Ce document a été généré automatiquement le 22 mai 2021

(c) Association Recherches en sciences sociales sur Internet 


\section{Du tritium dans les gros titres}

Rumeurs anxiogènes et remise en cause des médias sur Twitter

Tritium in the headlines. Alarming rumors and media blaming on Twitter

Antonin Segault

\section{Introduction}

1 Services de fact-checking dans les grands quotidiens nationaux, renforcement de l'éducation aux médias et à l'information dans les collèges, grandes enquêtes sur le complotisme... Les discours sur la qualité de l'information ont pris une place centrale dans l'espace public. Les incidents ayant émaillé, en 2016, l'élection de Donald Trump à la présidence des États-Unis et le vote du référendum pour la sortie du Royaume-Uni de l'Union Européenne auraient marqué l'avènement d'une ère de la "post-vérité " (Oxford Dictionaries, 2016), dans laquelle les faits auraient moins d'importance que les émotions ou les opinions personnelles, entraînant la prolifération d'informations erronées, biaisées ou tout bonnement fictives. D'autres décrivent un « âge de Twitter » (Ott, 2017) qui, après les âges de la typographie et de la télévision, serait caractérisé par la simplicité, l'impulsivité et l'incivilité des discours qui y circulent, dont archétype serait l'usage de Twitter par le président Trump. L'apparition simultanée et l'usage proche de ces deux expressions, «âge de Twitter » et " ère de la post-vérité », semblent impliquer l'existence, au moins dans l'imaginaire collectif, d'un lien voire d'une relation de causalité entre médias sociaux et informations douteuses. Une importante couverture médiatique, mais également de nombreux travaux scientifiques, ont ainsi été consacrés à la manière dont les médias sociaux affectent et sont affectés par la circulation d'informations erronées, le plus souvent sans que ce qualificatif ne soit questionné.

2 Cet article s'appuie sur les fondements de la sociologie de la déviance pour décrire les mécanismes de production, de circulation et de dénonciation des informations erronées sur Twitter. À travers l'analyse d'une rumeur portant sur la contamination radioactive de l'eau potable par du tritium durant l'été 2019, il montre que les modalités de gestion des déviances informationnelles font elle-mêmes l'objet d'une 
controverse, témoignant de la crise que traversent actuellement les médias, les experts et les institutions historiquement garantes de la vérité. Nous reviendrons tout d'abord sur les caractéristiques des médias sociaux, la manière dont les informations erronées s'y développent, et l'apport de la sociologie de la déviance pour en étudier la dénonciation (partie 1). Nous présenterons ensuite le cas d'étude, la controverse dans laquelle il s'inscrit et le corpus de données étudiées (partie 2). À l'aide de ce corpus, nous décrirons le cheminement suivi par la rumeur sur Twitter, de sa naissance à sa répression (partie 3), puis montrerons comment ce processus témoigne et contribue à la remise en cause de la place des médias dans la définition et l'application des normes de vérité (partie 4).

\section{Étudier les rumeurs sur les médias sociaux}

3 Les médias sociaux peuvent être définis comme des plateformes favorisant la production et la diffusion de contenus par les utilisateurs eux-mêmes (Kaplan \& Haenlein, 2010). Ce terme s'applique ainsi à un large spectre de dispositifs, allant des blogs personnels aux sites de réseaux sociaux tels que Facebook, en passant par les plateformes de partage de vidéos comme YouTube. Cet article s'intéressera particulièrement à Twitter, une plateforme de micro-blogging permettant la publication de courts messages (tweets) pouvant inclure des liens hypertextes, des contenus multimédias, des mentions d'autres utilisateurs, ou encore des mots clefs (hashtags) utilisés à des fins d'indexation. En abaissant les barrières techniques à la publication en ligne, de tels outils ont permis le développement d'un "web social ", plus participatif que le web des années 1990 (Breslin, Passant, \& Decker, 2009 : 12). Les usagers de ces dispositifs, contournant les gatekeepers traditionnels, ont ainsi obtenu un accès inédit à l'espace public : «Sur Internet, toutes les opinions se voient reconnaître le droit de cité par défaut, puisque tous les points sur le réseau sont techniquement à la même échelle» (Badouard, 2017: 91). Cette démultiplication des points de vue s'accompagne de phénomènes de diffusion virale (Heimbach, Schiller, Strufe, \& Hinz, 2015) pouvant se déployer à travers plusieurs plateformes en même temps (Segault, 2018). Une grande variété de discours sont par conséquent susceptibles de connaître une diffusion massive par le biais de ces outils.

4 Évidemment, les rumeurs, canulars, mensonges, et autres légendes urbaines qui circulent dans nos sociétés ont également trouvé leur place sur les médias sociaux. Certaines études ont d'abord laissé penser que le grand nombre d'usagers des plateformes de médias sociaux telles que Twitter permettrait le développement de processus collectifs de détection et de correction d'erreurs, favorisant la diffusion d'informations fiables (Mendoza, Poblete, \& Castillo, 2010; Sutton, Palen, \& Shklovski, 2008). En effet, de tels exemples d'une « sagesse des foules " (Surowiecki, 2004) ont déjà été constatés sur le web social, à l'image de l'encyclopédie contributive Wikipédia, dont la qualité résulte d'un processus de vigilance participative (Cardon \& Levrel, 2009). Mais des travaux plus récents ont montré que, sur Twitter, les informations erronées se diffusent plus rapidement que des informations vérifiables (Vosoughi et al., 2018), tout $\mathrm{du}$ moins lorsqu'elles proviennent de certains sites d'information (Bruns \& Keller, 2020). Il apparaît que certaines fonctionnalités et affordances des plateformes ellesmêmes sont susceptibles de contribuer à une amplification des rumeurs. Les algorithmes de recommandation de contenus pourraient notamment amplifier la 
diffusion de messages sensationnalistes et "conspirationnistes", dans la mesure où ceux-ci suscitent davantage de réactions que des contenus plus consensuels (AlgoTransparency, s.d.; Marwick, 2018). La circulation transmédia des messages donnerait également lieu à des phénomènes de «blanchiment de contenus », par lesquelles des informations douteuses sont successivement reprises par des sources de plus en plus crédibles (Frau-Meigs, $2019: 56$ ).

5 Au cours des dernières années, le rôle qu'ont joué ces formes de malinformation dans le déroulement de plusieurs événements majeurs (élections, attentats, etc.) a suscité une grande attention politique, médiatique et scientifique, voire un certain degré de "panique médiatique » (Frau-Meigs, 2019: 10). Le terme "fake news ", très largement employé dans ce contexte, recouvre en réalité une grande variété de situations, masquant notamment la différence entre les informations volontairement erronées, partagées sciemment par des personnes conscientes de leur caractère erroné, et le partage accidentel de contenus erronés par des personnes de bonne foi (Marwick, 2018). La notion d'information erronée est elle-même diverse : on peut distinguer les parodies, les contenus trompeurs, les impostures, les contenus fabriqués, les connexions fallacieuses, les contextes fallacieux ou encore les contenus manipulés (Wardle, 2017). Par ailleurs, le partage d'informations erronées, en particulier autour de thématiques politiques, s'inscrit parfois dans des logiques d'affichage idéologique accordant peu d'importance à la véracité des contenus : «ce qui importe n'est pas la justesse de l'information partagée, mais l'identité qu'elle signale" (Marwick, 2018, traduction personnelle) ${ }^{1}$. De fait, la complexité et la multiplicité des phénomènes que recouvre l'étiquette «fake news » constituent un important obstacle à la mise en œuvre de stratégies de luttes, mais aussi d'études scientifiques.

6 L'interactionnisme symbolique est fondé sur l'idée que les personnes n'agissent pas tant en fonction des actions des autres que selon la signification qu'elles attribuent à ces actions (Blumer, 1962 : 91). Cette approche s'avère intéressante pour l'étude des phénomènes qualifiés de "fake news", car elle permet de s'affranchir de l'épineuse question de la véracité des énoncées pour se concentrer sur la manière dont ils sont qualifiés par les personnes qui les commentent. La rumeur, par exemple, peut être définie comme "l'émergence et la circulation dans le corps social d'informations, soit non encore confirmées publiquement par des sources officielles, soit démenties par celle-ci » (Alloing \& Vanderbiest, 2018; Kapferer, 1987). Cette formule montre bien que la qualification d'un énoncé comme véridique ou erroné est le résultat d'un processus d'évaluation par des acteurs dont l'avis est considéré comme valable dans un groupe donné : «ce qui définit la rumeur n'est pas la fausseté du contenu mais le fait que l'information va à l'encontre de l'information officielle " (Dauphin, 2019). Ainsi, par analogie avec les travaux d'Howard Becker sur la construction sociale de la déviance, la rumeur peut se définir comme un acte déviant, accusé de transgresser une certaine norme de vérité (Becker, 1985 : 32-33). L'étude de l'émergence et de la propagation des rumeurs nécessite par conséquent de s'intéresser au processus par lequel un discours donné est progressivement qualifié de rumeur et voit sa circulation évoluer face à la sanction sociale. Nous nous efforcerons ici de déterminer dans quelle mesure les médias sociaux affectent le déroulement et les résultats de ce processus. 


\section{L'alerte au tritium et ses tweets}

7 Dans cet article, cette problématique est abordée à travers un cas d'étude récent : la rumeur d'une dangereuse contamination radioactive de l'eau potable, qui a circulé sur les médias sociaux durant la seconde moitié du mois de juillet 2019. Il convient tout d'abord de rappeler le contexte ancien et complexe dans lequel cette rumeur a pu naître, puis de détailler le corpus utilisé pour son étude et les modalités de sa collecte.

\section{La réactivation d'une controverse socio-scientifique}

8 En France, l'énergie nucléaire tient une place prépondérante dans la production d'électricité. Elle rencontre néanmoins une opposition durable, liée à ses racines militaires et à sa gestion opaque, plus tard renforcée par les accidents et les défaillances ayant émaillé l'histoire de son exploitation (Perko et al., 2012; Topçu, 2006). Le risque radiologique, résultant de l'exposition aux rayonnements ionisants issus des matériaux radioactifs, est perçu comme particulièrement dangereux dans la population, notamment en raison de son invisibilité, de son origine humaine et de son caractère incontrôlable (Ropeik, 2008; Slovic, 1996). De fait, si certaines questions relatives aux risques radiologiques font l'objet de controverses scientifiques dans des milieux académiques, d'ordinaire relativement confinées (Lemieux, 2007), celles-ci se transforment souvent en "controverses socio-scientifiques", propulsées dans la sphère publique, soumises au regard et parfois à l'arbitrage des citoyens (Di Scala, 2019 : 13). Certaines controverses non-résolues, comme celle relative aux effets des faibles doses de rayonnements ionisants, sont régulièrement réactivées et remobilisées en réaction à des événements d'actualité ; on peut parler de " controverses à rebond " (Boudia, 2007). Au cours des dernières années, ces controverses se sont fréquemment exprimées sur les médias sociaux, en réaction aux projets d'enfouissement de déchets nucléaires (Badouard \& Mabi, 2015) ou à l'occasion d'un accident survenu dans une centrale (Segault et al., 2017).

Durant l'été 2019, le nucléaire a de nouveau fait l'actualité, suite à la publication par l'ACRO (Association pour le contrôle de la radioactivité dans l'ouest, créée au lendemain de l'accident de Tchernobyl pour assurer une surveillance citoyenne des centrales françaises), le 17 juillet, d'un rapport signalant une contamination de l'eau courante par du tritium. Cet isotope radioactif de l'hydrogène, produit par les centrales nucléaires, est signalé à des taux supérieurs à la normale (mais toujours significativement inférieurs aux seuils sanitaires) en de nombreux points en France. L'information, relayée en premier lieu dans un article du Canard Enchaîné, connaît ensuite une diffusion massive à travers des articles publiés sur les sites de grands quotidiens nationaux, largement relayés sur les médias sociaux. Quelques jours plus tard, des messages alarmistes sont partagés de manière virale, notamment via Snapchat et Facebook Messenger, appelant à ne pas boire l'eau courante en région parisienne en raison de sa contamination radioactive. En plein épisode de canicule, ces injonctions suscitent à leur tour de multiples démentis et clarifications de la part des hôpitaux, des autorités locales, de l'ACRO elle-même, et de nombreux organes de presse. Le procès en "fake news" qui couvait dès les premiers jours se déchaîne alors, dans les grands quotidiens comme sur les médias sociaux. 


\section{Constitution d'un corpus de tweets}

Cet article propose d'étudier cette séquence médiatique sur la base d'un corpus de tweets. Bien que Snapchat et Facebook Messenger aient joué un rôle clef dans la diffusion de messages alarmistes, ces deux plateformes, conçues pour l'échange de messages privés entre un nombre restreint d'utilisateurs, ne se prêtent guère à l'observation et au recueil de données. Twitter, à l'inverse, propose un riche écosystème d'interfaces de programmation (API) permettant la collecte de large corpus de messages et de leurs métadonnées (Bruns \& Stieglitz, 2012). Par ailleurs, cette plateforme présente la particularité d'avoir été employée tout au long de l'événement étudié, par un grand nombre d'acteurs (institutionnels et particuliers, pronucléaires et antinucléaires), et pour la diffusion de messages très variés (alarmistes, rassurants, ironiques, insultants, etc.). Elle offre donc un point de vue certes partiel mais assez transversal sur les échanges relatifs à la rumeur. Les discussions ayant eu lieu sur les autres plateformes ne pourront en revanche faire l'objet que d'imprécises conjectures.

11 Le corpus étudié a été constitué en interrogeant l'API REST Standard Search (Twitter, 2020b) à l'aide de la librairie logicielle Twython. Cette API permet la collecte des tweets contenant un mot clef donné (de manière non-exhaustive, mais Twitter n'indique pas selon quels critères la sélection est effectuée), sur une période pouvant s'étendre jusqu'à sept ou huit jours dans le passé. Ici, la requête portant sur le mot clef «tritium » a été lancée le 25 juillet 2019 en fin de soirée et l'API a fourni des messages remontant jusqu'au matin du 16 juillet. Un total de 23314 tweets a ainsi été collecté, dont 5144 tweets originaux (c'est-à-dire en excluant les retweets). La distribution temporelle des tweets (Figure 1) semble indiquer que ce corpus capture bien la période d'activité autour du mot clef, depuis les premiers soubresauts du 16 juillet, jusqu'à une diminution progressive de l'activité le 25 juillet.

En raison de l'urgence dans laquelle cette collecte a été paramétrée (afin d'éviter de "perdre» les tweets des premiers jours), certaines données capturées se sont finalement avérées incomplètes. En particulier, dans le cas des tweets longs de plus de 140 caractères, seule une version tronquée du message (ainsi que de ses hashtags, mentions, etc.) a été enregistrée. Pour y remédier, une nouvelle collecte de données a été effectuée le 6 octobre, afin d'actualiser les données (notamment le texte complet) de chaque tweet présent dans le corpus, un processus nommé "réhydratation " dans les documentations de Twitter (Twitter, 2020a). Cependant, en l'espace de deux mois, certains tweets n'étaient plus accessibles et n'ont pas pu être réhydratés, parce que leurs auteurs les avaient supprimés ou qu'ils avaient désactivé ou verrouillé leurs comptes. Un nombre considérable de retweets ont notamment disparu du corpus (Tableau 1). Des utilisateurs peuvent avoir dé-retweeté un message mais, pour la plus grande partie, il s'agit d'un artefact de la plateforme : quand un tweet est supprimé, tous ses retweets le sont automatiquement.

Tableau 1. Composition du corpus de tweets étudié

\begin{tabular}{|l|l|l|}
\hline Nombre de tweets & $\begin{array}{l}\text { Corpus initial } \\
\text { (tweets tronqués) }\end{array}$ & $\begin{array}{l}\text { Corpus réhydraté } \\
\text { (tweets complets) }\end{array}$ \\
\hline
\end{tabular}




\begin{tabular}{|l|l|l|}
\hline $\begin{array}{l}\text { Total } \\
\text { (retweets inclus) }\end{array}$ & 23314 & 18705 \\
\hline $\begin{array}{l}\text { Originaux } \\
\text { (retweets exclus) }\end{array}$ & 5144 & 4905 \\
\hline
\end{tabular}

Figure 1. Distribution temporelle des tweets du corpus

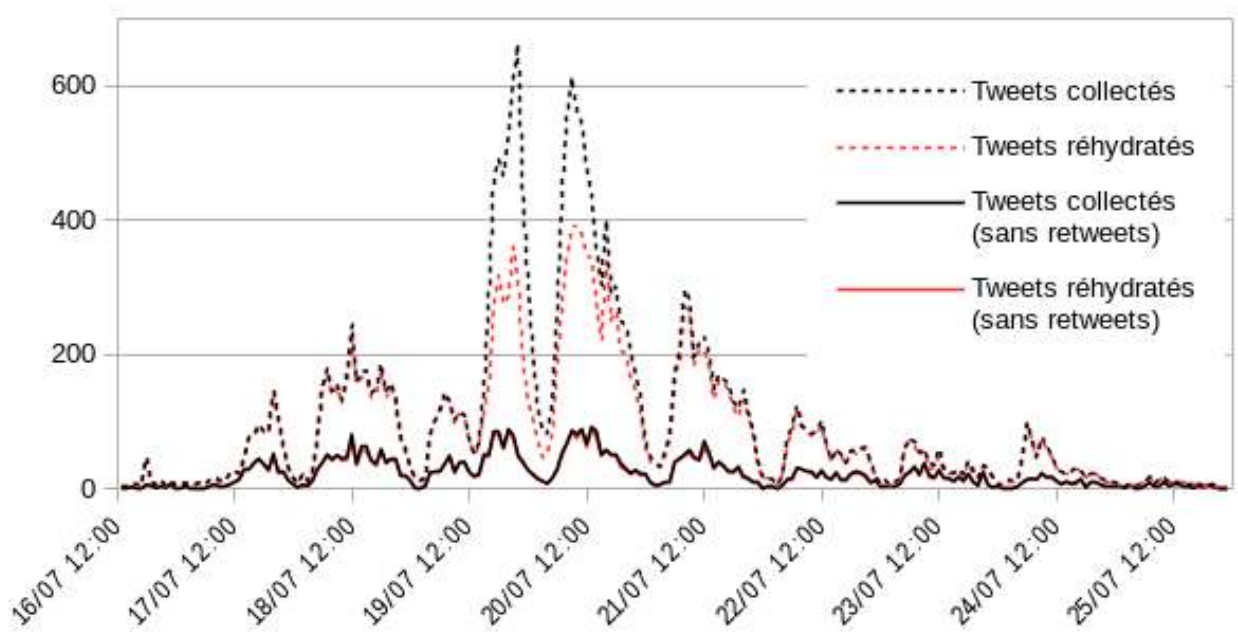

13 Ainsi, les analyses réalisées dans cet article portent sur un corpus hybride : une partie des tweets ont été réhydratés et comportent donc des métadonnées complètes, tandis que d'autres, supprimés avant l'étape de réhydratation, restent tronqués. Cette anomalie est susceptible d'affecter les résultats de certains calculs (par exemple si l'on compte le nombre de tweets contenant un terme ou un hashtag donné) mais s'est également avérée profitable pour l'étude. En effet, elle permet d'identifier et d'étudier les messages qui ont été publiés puis supprimés par leur auteur, ce qui n'est que rarement possible et pourtant très intéressant. Cependant, la retranscription de messages supprimés est éthiquement discutable, et ne saurait par conséquent être réalisée que dans des conditions limitant au maximum les désagréments potentiels pour leurs auteurs. Si les tweets sont généralement considérés comme des prises de paroles publiques, pouvant être librement reproduites, une grande majorité des usagers de Twitter souhaiteraient en réalité que leurs publications soient anonymisées lorsqu'elles sont exploitées dans le cadre d'études (Williams et al., 2017). Dans ce cas particulier, nous avons donc pris le parti d'anonymiser l'ensemble des tweets (supprimés ou non) issus des comptes tenus par des particuliers, en ne laissant visibles que les noms des comptes associés à des institutions (associations, partis politiques, collectivités locales, médias, etc.).

\section{Naissance et mort d'une rumeur en ligne}

14 Tout comme la déviance est « la conséquence de l'application, par les autres, de normes et de sanctions » (Becker, 1985 : 32-33), la qualification de rumeur peut être considérée comme le résultat d'un processus collectif par lequel un message, jugé problématique au regard d'une certaine norme, est progressivement désigné comme indésirable. 
L'examen de ce processus permet d'éclairer les différents éléments en jeu : les messages qui sont dénoncés, les normes qui sont invoquées, les modalités d'application de cette décision et les effets qu'elles engendrent sur la circulation des messages. On peut ainsi dessiner une image des arrangements sociaux au sein desquels la rumeur est produite et combattue.

\section{Émergence d'un message problématique}

La première trace de tritium dans le corpus étudié est un tweet du groupe écologiste du Conseil régional Centre-Val de Loire, publié dans l'après-midi du mardi 16 juillet :

«[RADIOACTIVITÉ DE LA LOIRE] L' @__ACRO__a indiqué une concentration de tritium dans les eaux de la Loire. Les élus écologistes de @Elus_EELV_PdL @ElusEcolosRCVL et @ElusEELVNvleAqu interpellent le préfet ».

Accompagné de deux images de la lettre destinée au préfet, il mentionne également plusieurs comptes Twitter tenus par Europe Écologie Les Verts (EELV) ou certains de ses membres, au niveau régional ou national. Ce tweet, dans sa forme, est caractéristique des pratiques de prise à témoin qui définissent les controverses socioscientifiques: un des acteurs (ici le parti EELV) engagé dans une controverse scientifique (la pollution au tritium) décide de l'exposer en interpellant publiquement un autre acteur (le préfet de la région Centre-Val de Loire) (Di Scala, 2019). L'usage intensif du système de mention propre à Twitter (les noms d'utilisateurs précédés d'une arobase) et les quelques centaines d'abonnés (followers) du compte ElusEcolosRCVL ont offert une certaine visibilité au message. Celui-ci a ainsi été partagé (retweeté) par 39 personnes, augmentant à leur tour sa visibilité. Le message d'ElusEcolosRCVL a suscité quelques réactions pendant la soirée, mais le sujet ne s'est emballé que le lendemain matin. 


\section{ACROACRO \\ @_ACRO \\ Carte exclusive de la contamination en \#tritium de \\ l'eau potable : plus de 6 millions de français \\ concernés à cause des rejets des installations \\ nucléaires. Et en cas d'accident \#nucléaire grave, quelle mesures de protection ?}

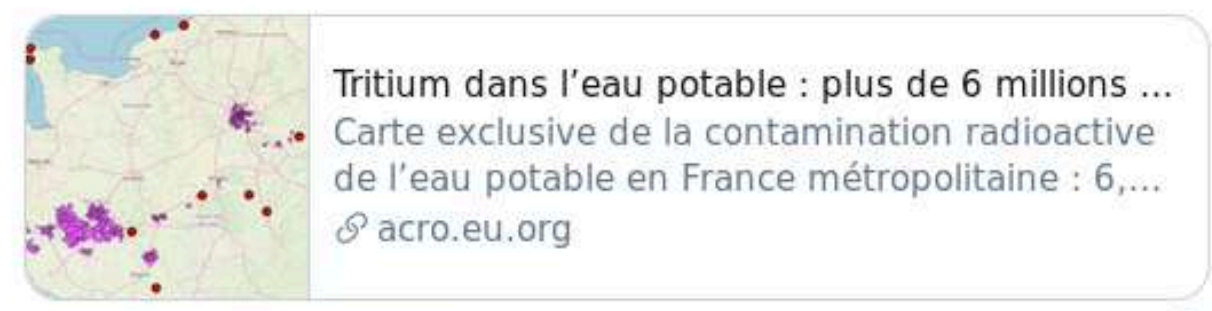

10:57 AM · Jul 17, 2019

\section{2}

\section{4 people are Tweeting about this}

Le mercredi 17 juillet, à partir de $9 \mathrm{~h}$, l'ACRO publie une série de tweets pointant vers une page de leur site "Tritium dans l'eau potable: plus de 6 millions de français concernés. Quelle eau potable en cas d'accident nucléaire grave? ", où se trouvent notamment une carte des mesures de tritium et un communiqué détaillé de six pages. Le premier de ces tweets (et le plus partagé, avec 56 retweets) adopte une formulation assez similaire : «Carte exclusive de la contamination en \#tritium de l'eau potable : plus de 6 millions de français concernés à cause des rejets des installations nucléaires. Et en cas d'accident \#nucléaire grave, quelles mesures de protection? (Figure 2). Un autre fait référence au Canard Enchaîné, dont l'édition du jour aborde le sujet, et un dernier, à 11h56, indique qu'une dépêche a été publiée par l'AFP sous un titre plus concis « Du \#tritium dans l'eau potable de millions de personnes (association) ». À partir de midi, le contenu de cette dépêche est repris, sous différents titres, par plusieurs médias à forte audience (Europe $1^{2}$, Le Parisien ${ }^{3}$, réseau France Bleu ${ }^{4}$, etc.). Les tweets et articles publiés par ces médias, ainsi que les discours d'accompagnement qui leur sont associés (Simon et al., 2017), occupent alors une place centrale dans la diffusion de l'information au cours des jours suivants.

Un article du Parisien intitulé «Du tritium décelé dans l'eau potable de 6,4 millions de personnes » est partagé de manière importante : son adresse URL apparaît dans plus de 60 tweets, dont certains sont largement retweetés (plus de 500 fois pour le tweet du Parisien lui-même). Un message contenant deux captures d'écran de cet article connaît une diffusion particulièrement massive après sa publication, le vendredi 19 juillet en début d'après-midi : «L'EAU DU ROBINET EST POLLUÉ PAR UN ÉLÉMENT RADIOACTIF (TRITIUM) DANS L'ÎLE DE FRANCE NE BUVEZ PAS ET PRÉVENEZ VOS PROCHES !!» (Figure 3). Ce message est le plus retweeté du corpus, avec 3718 rediffusions enregistrées (près de $16 \%$ des 23314 tweets du corpus). Sa tonalité alarmiste semble 
faire écho à un message audio circulant de proche en proche sur Facebook Messenger et Snapchat depuis la veille, dans lequel une personne (prétendant travailler aux Hôpitaux de Paris et disposer d'information émanant de la préfecture) incite les habitants d'île-de-France à ne pas boire d'eau du robinet. De nombreux autres tweets, moins largement partagés, adoptent un propos similaire : par exemple «L'eau de l'île de France et de paris sont contaminés au tritium c radioactive cancérigène, donc ne surtout pas boire l'eau du robinet, même après cuisson parce qu'on est pas encore sûr qu'il ne reste pas de substances radioactives. " (compte sous pseudonyme, datant de quelques mois et désormais bloqué, peu suivi) ${ }^{5}$. Ainsi, en quelques jours, les messages relatifs à la controverse socio-scientifique, d'abord virulents mais néanmoins nuancés, ont ensuite laissé place à des injonctions alarmistes sans équivoque.

Figure 3. Tweet alarmiste le plus diffusé (capturé sur Internet Archive en raison de sa suppression par son auteur).

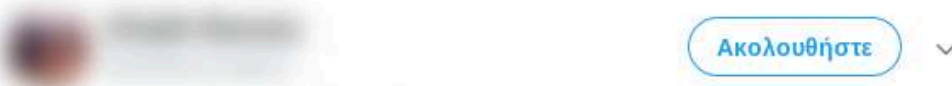 \\ L'EAU DU ROBINET EST POLLUÉ PAR UN ÉLÉMENT RADIOACTIF (TRITIUM) DANS L'ÎLE DE FRANCE NE BUVEZ PAS ET PRÉVENEZ VOS PROCHES !!}

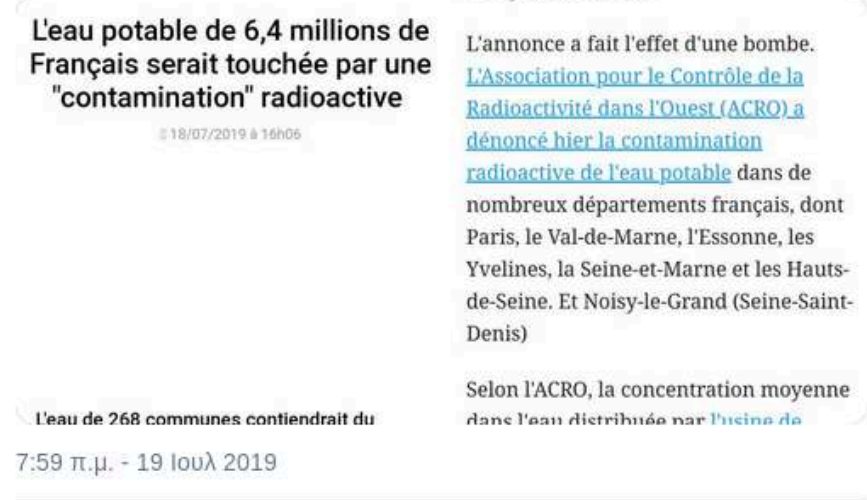

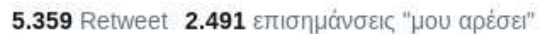

\section{Définition comme rumeur}

19 La diffusion de ces messages alarmistes a provoqué une réaction rapide d'un grand nombre d'acteurs institutionnels. Le vendredi 19, en début de soirée, un démenti est publié par la Préfecture de Paris : «Démenti sur la présence de \#tritium dans l'eau potable: aucun arrêté préfectoral n'a été pris par le préfet de Paris. Les valeurs observées à ce jour ne

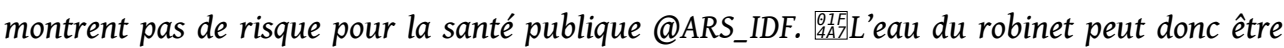
consommée sans restriction", retweeté près de 1500 fois. On trouve des messages similaires provenant d'autres acteurs locaux, tels que les préfectures des Yvelines et de Hauts-de-Seine (Figure 4), la mairie de Trappes, mais aussi d'experts, comme la régie Eau de Paris et les Hôpitaux de Paris. L'ACRO publie également un message de clarification dans la soirée du vendredi : "Quelques messages circulent sur les réseaux 
faisant état d'une interdiction de consommer de l'eau dans les hôpitaux parisiens. C'est complètement faux. Comme nous l'avons dit, aucune valeur ne dépasse le critère de qualité fixé à100 Bq/L. ». Un consensus semble ainsi s'être établi, chez les autorités, chez les experts, mais aussi chez les militants écologistes, autour de l'inexactitude du message appelant à cesser la consommation de l'eau du robinet.

Figure 4. Tweet de la Préfecture de Hauts-de-Seine, visant à rassurer les habitants quant à la potabilité de l'eau, chiffres à l'appui.

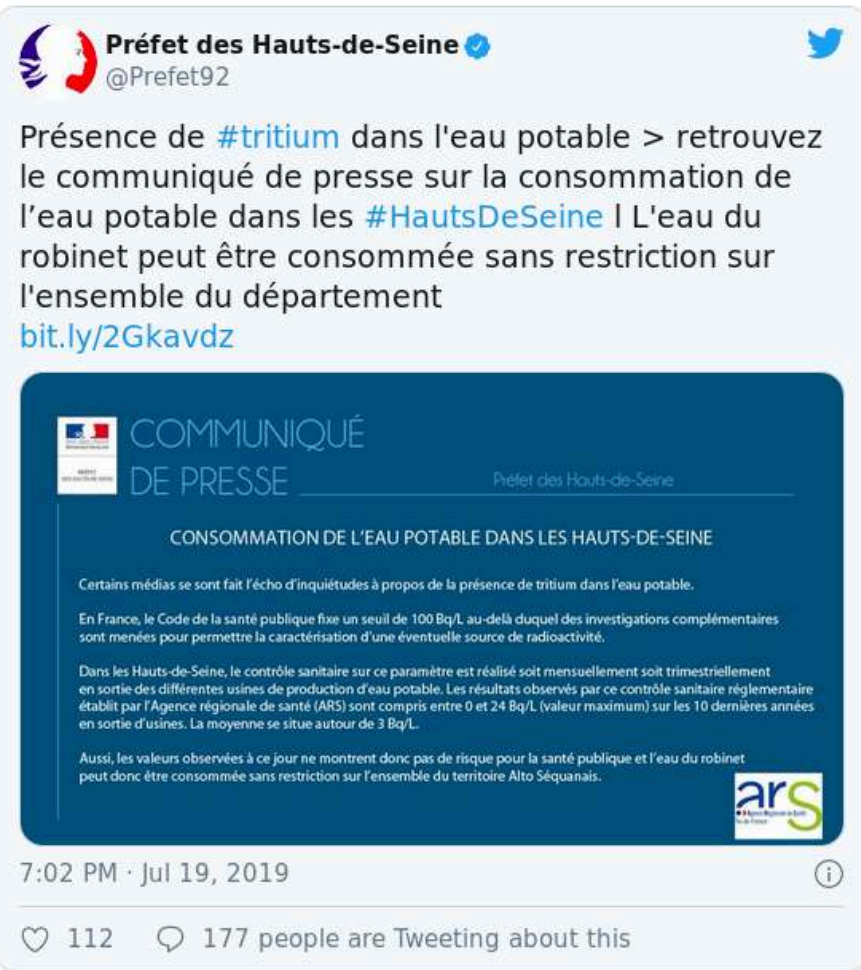

À partir du samedi 20 (midi), une nouvelle série de tweets sont alors publiés par les mêmes médias nationaux, reprenant les démentis publiés la veille au soir : «Arrêter de boire de l'eau du robinet à cause du tritium? La préfecture dément et rassure " (L'Express). Certains adoptent une forme interrogative, questionnant le danger de la consommation d'eau, comme BFM TV: «Rumeurs de pollution au tritium: l'eau du robinet présente-t-elle vraiment un risque en Île-de-France? », ou encore France Info : «VRAI OU FAKE La présence de tritium, une substance radioactive, dans l'eau potable est-elle dangereuse pour votre santé?». Mais les titres affirmatifs, assimilant le message à une rumeur ou à une "fake news ", se généralisent progressivement dans le traitement médiatique, comme dans ce tweet fortement partagé du Parisien: «Eau de Paris 'polluée' au tritium: gare aux fake news ». Une série de messages du compte Conspiracy Watch (tenu par l'association Observatoire du conspirationnisme) va dans le même sens: "Les \#rumeurs sur une prétendue contamination de l'\#EauPotable au \#tritium vont bon train depuis hier. Colportées notamment par une personne se faisant passer pour une infirmière de l'hôpital Bichat à Paris. ».

21 Si les messages alarmistes ont commencé à circuler dès le jeudi 18 juillet, ce n'est que dans la journée du samedi 20 que le qualificatif de rumeur leur a été publiquement attribué. Tant que la critique se faisait dans des cercles restreints, au gré des partages sur les plateformes de discussion, et donc de manière discrète, les messages restaient 
globalement épargnés par la pression sociale. Tout au plus, pouvait-on reprocher à l'expéditeur un manque de recul critique sur une information s'appuyant sur des sources douteuses. Mais à partir du moment où ces messages ont fait l'objet d'une condamnation unanime par les experts de tous bords, largement diffusée sur les médias nationaux, leur caractère fallacieux est devenu à la fois incontestable et immanquable. L'utilisation des termes "rumeur » ou "fake news ", parfois sous forme de hashtags, de même que l'intervention de Conspiracy Watch sont autant de marques d'infamie, en raison de la connotation fortement négative associée à ce vocabulaire. Le verdict est clair : ces messages sont contraires à la norme de vérité en vigueur et leur partage constitue une déviance qui doit être sanctionnée.

\section{Pressions et conformations}

Le statut de la rumeur ayant été publiquement établi, de nombreux utilisateurs publient des tweets à destination des personnes qui ont participé à sa diffusion. Certains tentent de présenter de manière pédagogique les niveaux de radioactivité dont il est question. Une image fréquemment partagée compare les doses de rayonnements reçues en buvant l'eau du robinet et en mangeant une banane, fruit présentant une légère radioactivité naturelle, due à un isotope radioactif du potassium (Figure 5). D'autres tweets visent à rétablir les nuances originales de la publication de l'ACRO :

«l'exposition au tritium, aux doses observées, ne soulève pas la moindre inquiétude chez les spécialistes de la radiotoxicité. Pas-la-moindre. [...] EN REVANCHE, oui, il faut mesurer le tritium, et s'alarmer d'une variation des concentrations relevées elles peuvent révéler une fuite, un manquement, un défaut d'étanchéité des centrales» (compte de journaliste, impliqué dans plusieurs autres controverses socio-scientifiques, très suivi).

Des messages d'explication sont publiés en réponse à certains des tweets alarmistes présentés plus haut :

«Le pain que l'on mange est à plusieurs centaines $\mathrm{de} \mathrm{Bq} / \mathrm{kg}$, le cas du tritium c'est surtout bien pour qu'on réfléchisse à ce qui se passerait en cas de soucis. Fais attention, ton tweet est plus dangereux que la contamination car une fausse alerte peut entraîner des paniques $\wedge^{\wedge}$ » (compte sous pseudonyme, contenus principalement liés à la pop-culture, assez suivi). 
Figure 5. Une infographie pédagogique visant à contrer la rumeur (initialement publiée par le compte d'un auteur très suivi, « passionné de sciences [...] et d'esprit critique » ; mais l'usage de la banane comme valeur de comparaison est bien plus ancienne ${ }^{6}$ )

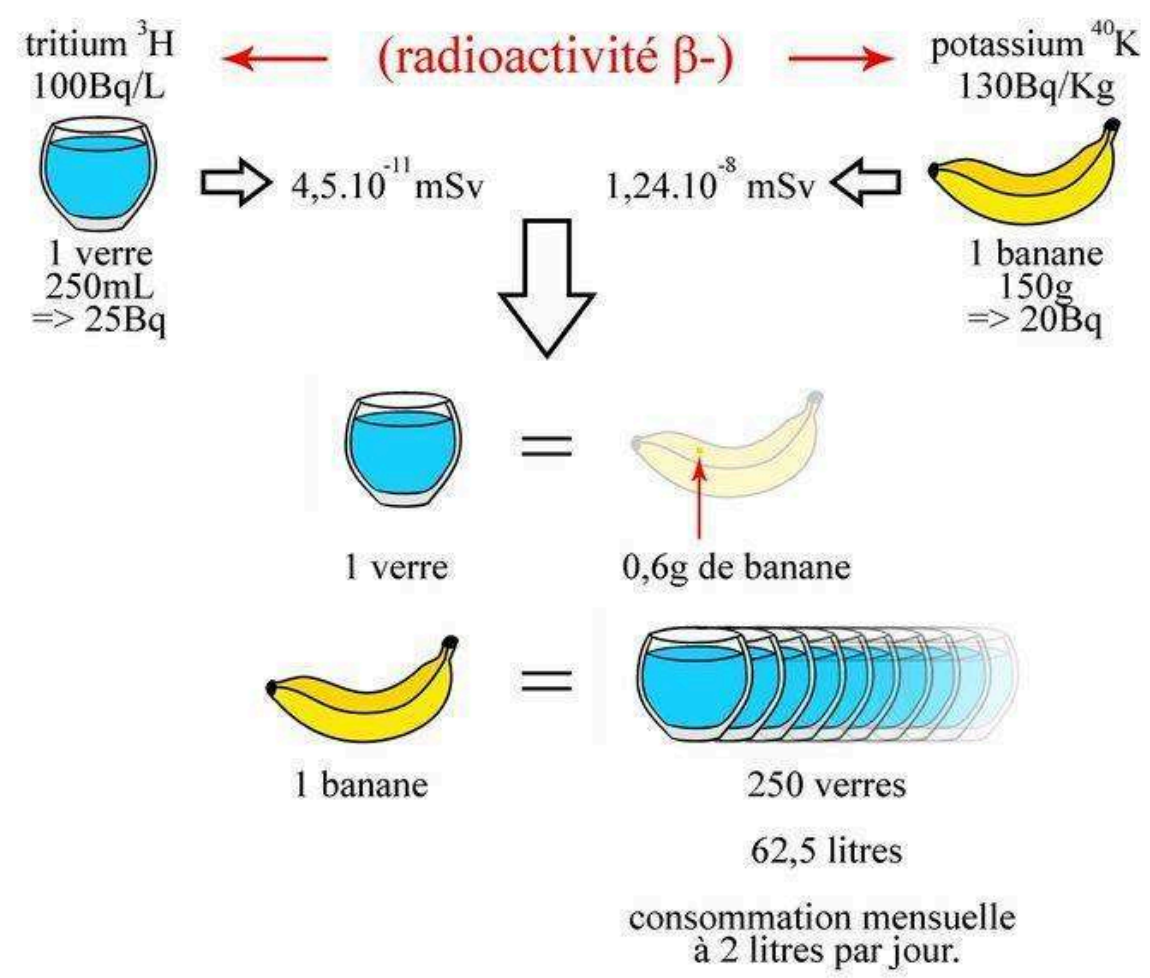

Néanmoins, bon nombre des réponses envoyées sont nettement moins polies : «Sinon tu te renseignes 30 secondes sur ce qu'est le tritium et sur les taux à risque, et tu ne relaie pas de la merde " (compte sous pseudonyme, messages souvent politiques et critiques de l'action du gouvernement LREM, peu suivi). En effet, dans les flux de réponses, les discussions constructives entre les personnes ayant relayé les messages alarmistes et leurs détracteurs sont bien plus rares que les échanges de critiques voire d'insultes, témoignant de la «brutalisation du débat public » (Badouard, $2017: 65$ ). Face à l'ampleur et la virulence des accusations, plusieurs utilisateurs choisissent finalement de supprimer leurs messages incriminants. Si l'étude des tweets supprimés est généralement complexe, la procédure de réhydratation effectuée sur le corpus a permis de facilement identifier les messages qui ont été supprimés entre la date de collecte (le 25 juillet) et la date de réhydratation (le 6 octobre). On constate ainsi la disparition de plusieurs des tweets alarmistes, dont celui de la Figure 3 (la suppression de ce tweet, entraînant la disparition de tous ses retweets, explique à elle seule une grande part de la différence de volume entre les corpus initiaux et réhydratés). Le nettoyage de profils Facebook a déjà été mis en évidence, notamment chez les adolescents, dans le cadre de stratégies visant à reprendre le contrôle de sa vie privée, en évitant que des messages anciens ne restent visibles (Boyd, $2016: 144$; Raynes-Goldie, 2010). Ici, les suppressions semblent moins réalisées dans un but préventif, pour se protéger d'attaques potentielles, que curatif, en réaction à des critiques directes.

Les tweets envoyés aux personnes ayant contribué à la diffusion de la rumeur, qu'il s'agisse de messages pédagogiques ou plus virulents, partagent le même objectif : leur montrer qu'ils sont dans l'erreur et les y faire renoncer. Ils sont ainsi l'expression de la pression sociale qui s'exerce sur les auteurs d'actions catégorisées comme déviantes 
pour les contraindre à suivre la norme: "Placé pour la première fois devant les conséquences ultimes et brutales que son action risque d'entraîner, il peut décider qu'il n'est pas souhaitable de s'engager plus avant sur la voie de la déviance et rebrousser chemin. S'il fait le bon choix, son retour dans la communauté des gens qui respectent la norme sera bien accueilli» (Becker, 1985: 60). De fait, une fois que les auteurs reconnaissent leur tort, ils ne semblent plus guère inquiétés. La suppression des messages et la fin des brimades apparaissent, pour eux comme pour leurs accusateurs, comme le meilleur moyen de "préserver la face» (Goffman, 1974). Ainsi, la dénonciation publique de la rumeur par tous les acteurs précédemment évoqués semble-t-elle avoir créé un cadre normatif suffisamment ferme pour en assurer un rapide étouffement.

\section{Temporalités de la rumeur}

La description déployée dans les précédents paragraphes pourrait laisser penser que la rumeur a suivi une trajectoire rectiligne, au cours de laquelle des étapes successives de diffusion, de dénonciation et de répression s'enchaînent distinctement (Tableau 2). L'analyse des données montre cependant une temporalité plus floue, dans laquelle ces étapes présentent des transitions et des chevauchements plus ou moins longs. Ainsi, des messages alarmistes ont continué à être publiés et retweetés bien après la large médiatisation des démentis officiels. La Figure 6, qui compare la distribution temporelle des retweets du message alarmiste le plus partagé (présenté dans la Figure 3) et du démenti de la Préfecture de Paris, montre que le premier voit sa diffusion diminuer après la publication du second, mais reste encore partagé jusqu'au 22 juillet. De même, le dimanche 21, plusieurs utilisateurs partageaient encore des liens vers d'anciens articles de presse (relatifs à la contamination, mais pas à sa relativisation) ou encore de nouveaux messages à caractère alarmiste: «Urgent tritium urgent, ne pas boire l'eau du robinet à Paris et envir... » (compte sous pseudonyme, peu suivi, relayant souvent des vidéos dénonçant des complots ou des effets négatifs des vaccins ; message accompagné d'un lien vers une vidéo YouTube reprenant le message audio circulant sur Snapchat avec un commentaire du même ton). Ces partages résiduels, sans remettre en question le processus par lequel un message a été désigné comme rumeur et par conséquent réprimé, offrent un éclairage intéressant sur le déroulement de ce même processus.

Tableau 2. Présentation chronologique de quelques tweets clefs

\begin{tabular}{|c|c|c|}
\hline Date & Auteur & Tweet \\
\hline $\begin{array}{l}16 / 07 \\
14: 20\end{array}$ & $\begin{array}{l}\text { Groupe } \\
\text { Ecologiste }\end{array}$ & $\begin{array}{l}\text { [RADIOACTIVITÉ DE LA LOIRE }] \quad \text { L' @__ACRO_- a indiqué une } \\
\text { concentration de tritium dans les eaux de ... }\end{array}$ \\
\hline $\begin{array}{l}17 / 07 \\
08: 57\end{array}$ & ACRO & $\begin{array}{l}\text { Carte exclusive de la contamination en \#tritium de l'eau potable : plus } \\
\text { de } 6 \text { millions de français ... }\end{array}$ \\
\hline $\begin{array}{l}17 / 07 \\
15: 49\end{array}$ & Le Parisien & $\begin{array}{l}\mathrm{Du} \text { tritium, forme radioactive de l'hydrogène, retrouvé dans l'eau } \\
\text { potable de } 6,4 \text { millions de personnes ... }\end{array}$ \\
\hline
\end{tabular}




\begin{tabular}{|l|l|l|}
\hline 19/07 & & $\begin{array}{l}\text { L'EAU DU ROBINET EST POLLUÉ PAR UN ÉLÉMENT RADIOACTIF } \\
\text { (TRITIUM) DANS L'ÎLE DE FRANCE NE BUVEZ PAS ... }\end{array}$ \\
\hline $\begin{array}{l}\text { 19/07 } \\
18: 32\end{array}$ & Prefet75 IDF & $\begin{array}{l}\text { Démenti sur la présence de \#tritium dans l'eau potable : aucun arrêté } \\
\text { préfectoral n'a été pris ... }\end{array}$ \\
\hline $\begin{array}{l}\text { 20/07 } \\
11: 02\end{array}$ & Conspiracy & $\begin{array}{l}\text { Les \#rumeurs sur une prétendue contamination de l'\#EauPotable au } \\
\text { \#tritium vont bon train ... }\end{array}$ \\
\hline $\begin{array}{l}\text { 20/07 } \\
\text { 19:57 }\end{array}$ & Le Parisien & Eau de Paris «polluée» au tritium : gare aux fake news \\
\hline $\begin{array}{l}\text { 23/07 } \\
15: 22\end{array}$ & Reporterre & $\begin{array}{l}\text { Tritium dans l'eau : « Aucune raison de paniquer mais beaucoup de } \\
\text { raisons de se mobiliser » }\end{array}$ \\
\hline
\end{tabular}

Figure 6. Comparaison de la dynamique temporelle de partage du message alarmiste de la Figure 3 et du démenti publié par la Préfecture de Paris, montrant que la diffusion du second ne jugule pas directement celle du premier le premier.

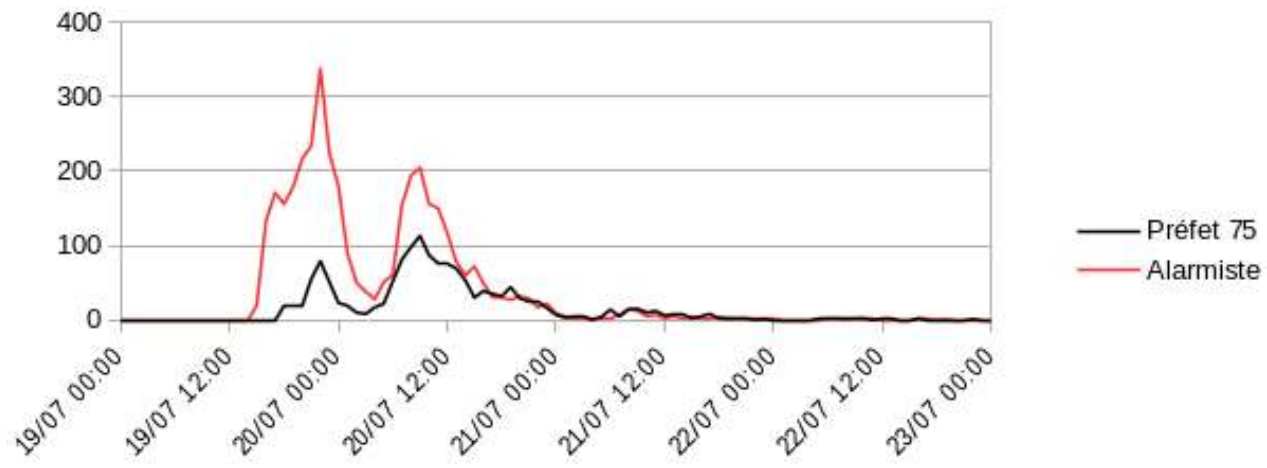

Plusieurs hypothèses peuvent être proposées pour expliquer ces chevauchements de messages contradictoires. Le grand nombre d'usagers de Twitter, combiné à la fragmentation idéologique et générationnelle qui s'y renforce (Hahn et al., 2015), peut tout d'abord induire une certaine inertie dans la dénonciation de la rumeur. En effet, certains utilisateurs peuvent, en raison de la composition de leur liste d'abonnements, ne pas avoir reçu ou vu les messages de démenti. Cet isolement peut être fortuit, dans le cas d'utilisateurs suivant peu de comptes ou des comptes liés à des thématiques très éloignés de l'actualité. Néanmoins, il peut aussi être le résultat de phénomènes d'homophilie, par lesquels se rassemblent des utilisateurs partageant un certain nombre de vues sur le sujet: "Des communautés d'opinions se forment de manière éphémère, autour de thématiques spécifiques, en prenant cependant soin de mettre à distance les opposants potentiels.»(Badouard, 2017: 81). Des îlots de résistance pourraient ainsi avoir subsisté au sein desquels, les messages de démentis ne circulant pas, la diffusion des messages alarmistes ne serait pas affectée par la pression sociale. Par ailleurs, des tweets mettant en doute les démentis semblent avoir participé à cette persistance: "Comment peut on affirmer qu il $\mathrm{n}$ y a aucun risque alors qu aucune étude sur les effets a long terme $\mathrm{n}$ a été mener \#transparence \#tritium @Prefet91 @ARS_IDF » (compte sous pseudonyme, indiquant des liens avec la Seine-Saint-Denis, peu suivi), ou encore «Bonjour @mairievitry malgré le démenti de @Prefet94 au sujet 
du \#tritium y a-t-il des dispositions prévues pour les centres de loisirs? \#principedeprecaution \#radioactivité @__ACRO_-" (compte d'un informaticien, indiquant des liens avec le Val-de-Marne, assez suivi). Ces messages se nourrissent de la défiance actuelle à l'égard des autorités et des médias (cf. infra), mais également du rapport historiquement conflictuel au risque radiologique: «@APHP @Paris @Prefet75_IDF le tritium bien sùr s'arrète à la frontière des robinet!» (compte sous pseudonyme, publiant rarement, peu suivi), en référence manifeste à la gestion de l'accident nucléaire de Tchernobyl.

La persistance des tweets alarmistes tient également à leur diffusion cross-media. De nombreux messages relatifs à la potabilité de l'eau ont circulé sur des plateformes de messagerie telles que Snapchat et Facebook Messenger. La nature de ces dispositifs empêchant la diffusion massive d'un démenti par les autorités ou les médias, le statut de la rumeur a certainement mis plus de temps à s'y imposer. Certains des tweets alarmistes apparus dans les jours suivant les démentis seraient alors des résurgences issues de ces plateformes, témoignant de cette différence de temporalité : tandis que la rumeur était déjà dénoncée et relativement étouffée sur Twitter, elle restait considérée comme crédible et donc partagée sur les messageries. Néanmoins, cette différence pourrait aussi être une conséquence de la pression sociale qui s'est fortement exercée sur Twitter après la dénonciation de la rumeur: "Au final, il découle de cette brutalisation générale du débat un phénomène d'évitement. Les internautes 'ordinaires' préfèrent fuir la conversation politique quand celle-ci a lieu sur des plateformes fréquentées par des individus aux positions trop divergentes pour se rabattre sur des sites orientés idéologiquement» (Badouard, 2017: 80). Ainsi, les personnes persistant dans la déviance informationnelle pourraient se retrouver dans des espaces en ligne spécifiques, comme on l'observe autour d'un certain nombre de sites «conspirationnistes.» Ce regroupement favoriserait alors la persistance de la rumeur, malgré la pression normative, mais aussi l'inscription de certains utilisateurs dans des "carrières informationnelles déviantes " à plus long terme : " une fois entré dans un groupe déviant organisé et institutionnalisé, le déviant a plus de chance de poursuivre dans cette voie. D'une part il a appris comment éviter les difficultés ; d'autre part, il a acquis un système de justification qui l'incite à persévérer » (Becker, 1985 : $62)$.

\section{Les conflits derrière la rumeur}

L'étude du développement de la rumeur met en lumière ses conditions d'émergence et d'évolution, dans un contexte social et technique donné. Mais elle révèle également de multiples groupes d'acteurs, aux intérêts variés, qui s'expriment autour de la question du tritium en la liant aux problématiques qui leur sont propres. Le traitement de la rumeur est ainsi traversé par des enjeux de pouvoir, s'inscrivant dans des conflits et des controverses plus larges et plus anciens.

\section{Controverse des spécialistes}

Si les messages alarmistes portant sur la consommation d'eau du robinet ont fait l'objet d'une condamnation franche et massive après la publication des démentis officiels, certains internautes dénoncent également d'autres messages relatifs au tritium. Dès les 
premières publications de l'ACRO, des tweets ont questionné la gravité de la contamination: " No fucking joke? Evidemment qu'il y a du deuterium et du tritium dans l'eau, ce sont des isotopes de l'hydrogène, il y en a partout, en quantité négligeable. » (compte d'un français expatrié dans le domaine de la finance, libéral et anti-communiste autoproclamé, très suivi). Ils accusent l'association de vouloir créer la panique: «@_ACRO_- 1/25000 de la dose limite réglementaire annuelle: c'est l'impact maximal du tritium ; alors pourquoi ces propos alarmistes? Pour faire peur, voilà la solution retenue par les militants antinucléaires. Malhonnête!» (compte d'un ingénieur dans le domaine de l'énergie, favorable au nucléaire, peu suivi). Plus généralement, ces détracteurs mettent en doute l'importance, voire la validité des faits dénoncés par l'ACRO, dont ils remettent cause l'expertise et l'intégrité. Ainsi, la controverse socio-scientifique à l'origine de la rumeur a-t-elle continué de se jouer, en parallèle et de manière bien moins visible, entre des factions de spécialistes.

Figure 7. Tweet interpellant plusieurs utilisateurs qui affichent leur appartenance ou leur proximité avec l'industrie nucléaire (les mentions, au début du tweet, sont floutées dans la capture).

\section{Avez-vous vu passer cet entretien et ses recommandations :}

\section{reporterre.net/Tritium-dans-I...}

\section{Un seuil de $2 \mathrm{~Bq} / \mathrm{L}$ *facepalm*}

La Criirad souhaite que la référence de qualité soit abaissée à terme de $100 \mathrm{~Bq} / \mathrm{L}$ à 2 $\mathrm{Bq} / \mathrm{L}$ et qu'elle serve à détecter toutes les contaminations par le tritium (et pas seulement par d'autres radionucléides). II faut les recenser, s'interroger sur leur origine, leur cause, leur durée et mettre en œuvre, le cas échéant, des actions correctives.

11:07 AM - Jul 25, 2019

Tout comme le tweet initial d'ElusEcolosRCVL mentionnait plusieurs comptes liés à l'écologie, vraisemblablement pour les inciter à rediffuser l'information, les critiques des messages de l'ACRO s'accompagnent de mentions visant à attirer l'attention de certains utilisateurs (Figure 7). Un même compte (ici nommé @XXXX) est interpellé à plusieurs reprises: «J'ai du mal avec l'accent alarmiste de l'article. Le tritium peut se trouver dans l'eau et ça permet même de dater un aquifère pour savoir à quelle vitesse il se recharge. Les valeurs retenues sont très en dessous des limites. Ca sent le FUD. @XXXX?» (compte d'un enseignant en sciences physiques, peu suivi) (FUD est l'acronyme de "fear, uncertainty and doubt", désignant les opérations visant à influencer le grand public en utilisant la peur), puis : «@XXXX tu as vu le communiqué de l'ACRO sur le tritium dans l'eau, lié sus rejets des centrales ce matin relayé par le 
canard enchaîné ?» (compte d'un micro-biologiste, publiant souvent sur des sujets relatifs à l'eau, assez suivi). Ce compte très suivi (@XXXX) se présente comme "Ingénieur sûreté et vulgarisateur (amateur) \#nucléaire", un autre indique être «Membre de @voixdunucleaire », une association pour la promotion du nucléaire, un troisième dit travailler dans la gestion des déchets nucléaires. On observe ainsi l'opposition des deux camps historiques de la controverse socio-scientifique sur le nucléaire: les écologistes anti-nucléaires contre les membres et soutiens de cette industrie.

Certains partisans de l'ACRO invoquent le principe de précaution face aux incertitudes quant aux effets des petites quantités de tritium: «Des études ont été faites sur la dangerosité du tritium à fortes doses, jamais sur les conséquences d'une absorption régulière de faibles doses sur le long terme... » (compte d'un militant pour les droits des peuples autochtones, assez suivi), tout en veillant à ne pas cautionner les messages alarmistes: "Personne n'a dit que l'eau n'était pas potable. Il faut lire le texte du communiqué. On parle d'une surdose anormale. Par contre, on peut s'interroger sur les effets potentiellement cancérigènes de l'exposition aux faibles doses de tritium dans l'eau potable » (compte du groupe écologiste du Conseil régional Centre-Val de Loire, assez suivi). La rumeur a ainsi suscité une réactivation de la controverse socioscientifique sur les faibles doses d'irradiation, qui effectue un nouveau "rebond" (Boudia, 2007). Les détracteurs de l'ACRO, de leur côté, préfèrent pointer vers d'autres risques, qu'ils jugent comparativement plus importants: "Puisque l'eau au tritium passionne, revoici la carte du radon en France. Le radon est un gaz radioactif, incolore et inodore, bien plus dangereux et pourtant bien naturel puisque issu de la désintégration du radium de la croûte terrestre, notamment dans le granite " (compte de journaliste, publiant souvent sur des sujets techniques, très suivi). Le radon étant d'origine naturelle, contrairement au tritium dont il est ici question, on peut y voir une tentative de déplacer le débat vers un domaine n'impliquant pas l'industrie du nucléaire.

Sur ces questions précises, les divisions historiques des deux camps se ravivent et la véracité de chaque affirmation fait débat. Le consensus, qui s'était si vite imposé autour de la potabilité de l'eau, semble cette fois impossible : «Les acteurs eux-mêmes ne sont pas d'accord sur ce qui est déviant et [ils] doutent souvent du caractère déviant de tel acte » (Becker, 1985 : 208). Chaque camp soupçonne l'autre de manipulation, voire de machination, au profit du lobby nucléaire : «Affaire du tritium dans l'eau : pourquoi ne cherche-t-on pas la fameuse "infirmière" qui raconte des sornettes? On a certainement peur de trouver qu'elle émarge au lobby pro nucléaire en caricaturant la recherche scientifique de l'ACRO. » (compte d'un enseignant-chercheur en SHS, très suivi), ou de son pendant anti-nucléaire: "Contamination au tritium des rivières françaises: anatomie d'une opération destinée à générer une peur infondée sur le nucléaire " (compte français du média en ligne Atlantico, très suivi). Les deux factions accusent les journalistes de faire de jeu de l'autre: "Les médias se laissent abuser par des associations qui sont toutes antinucléaires et dont le seul but est de faire le buzz. Récemment, on a inquiété les parisiens sur le niveau de tritium dans l'eau potable !» (compte sous pseudonyme, engagé pour le développement durable, peu suivi) ou encore : "Pourquoi les journalistes et les lobbyistes du nucléaire ont-ils détourné le rapport de son objet, alors qu'il spécifiait en gras, dès les premières lignes, que le taux de radioactivité était 10 fois plus petit que le seuil d'alerte? Fact checking. » (compte sous pseudonyme, collectif d'universitaires, proche de la gauche, très suivi). La 
controverse socio-scientifique, loin de trouver une résolution, semble se nourrir de chaque événement, laissant prévoir de nouveaux rebonds à l'avenir.

\section{Responsabilité de la rumeur}

En dépit des désaccords durables et non résolus, un consensus émerge cependant entre pro- et anti-nucléaires, sur un tout autre point. Dans un échange de messages avec l'ACRO, l'un des utilisateurs affiliés à l'industrie nucléaire indique être moins ennuyé par leur publication que par le traitement qu'en ont fait les médias : «Que ce soit clair, je ne vous reproche pas votre communiqué de presse. J'ai une interrogation sur la valeur des rejets de Nogent, mais pour le reste vous êtes dans votre "ligne editoriale". Ce que je reproche, c'est à @afpfr de ne pas l'avoir mis en perspective ». Comme cet extrait le suggère, certains acteurs de la controverse jugent ici que les médias, en ne leur permettant pas correctement de prendre le public à témoin du débat qui les oppose, manquent à leur devoir. Que les garants de la norme de vérité se rendraient eux-mêmes coupables de déviances informationnelles.

Figure 8. Représentation schématique des jeux d'acteurs des deux controverses visibles dans le corpus.
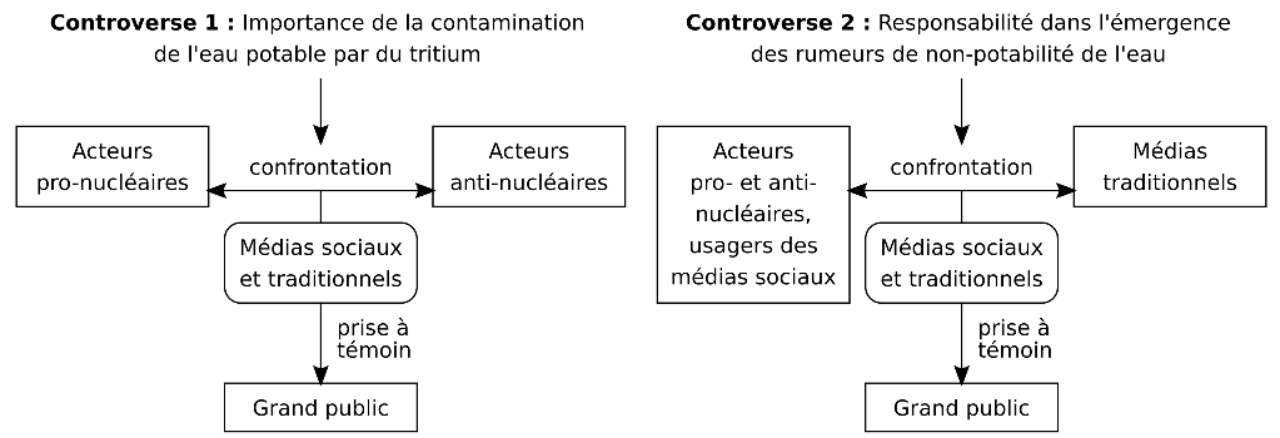

Une seconde controverse apparaît ainsi dans le corpus, non plus comme cause mais comme conséquence de la diffusion de la rumeur (Figure 8). Plusieurs des articles publiés par la presse après les démentis officiels désignent, plus ou moins directement, les médias sociaux comme responsables de la diffusion, voire de l'apparition des messages alarmistes. Le quotidien Sud Ouest titre : «Eau contaminée au tritium : d'où vient la rumeur qui circule depuis quelques jours sur les réseaux sociaux?». Ce discours est repris par de nombreux tweets, reflétant l'idée reçue d'un lien de causalité entre médias sociaux et information erronée : « cette histoire de tritium montre bien le pouvoir de la désinformation à $l$ air du tout partage via réseaux sociaux et le mal que cela peut engendrer... les gens relayent sans vraiment savoir sur la base $d$ une alerte ou $\mathrm{d}$ un mensonge faussement argumenté » (compte sous pseudonyme, publie sur le sport, les technologies, la pop culture, très suivi). Mais ces critiques déclenchent les foudres des utilisateurs des médias sociaux, dénonçant un double discours de la presse, d'abord alarmiste puis moralisateur : "Médias : Le TRITIUM ! Dans l'EAU ! On va tous mourir ! Quelques comptes proéminents sur le nets: Bah en fait non ça va. C'est large en dessous des seuils dangereux. Médias: Comment Internet a propagé la rumeur de la contamination de l'eau au Tritium. Enquête. » (compte sous pseudonyme, se décrivant comme libertarien, assez suivi). L'attribution de la responsabilité de la rumeur voit ainsi s'opposer les défenseurs de la presse et des médias sociaux dans une controverse 
au parfum de querelle des Anciens et des Modernes, dont l'expression sur Twitter est évidemment favorable aux médias sociaux.

De nombreux tweets reprennent les articles publiés avant le démenti officiel pour montrer les faiblesses du traitement de l'information : « Aujourd'hui à @Europe1 : Dis il y a un truc qui a l'air vachement flippant sur le tritium radioactif. Super écris le bien gros! Bon en fait il disent que c'est pas grave du tout. Ah mince... bah met le en tout petit dessous alors, faut pas trop que ça se voit » (compte sous pseudonyme, engagé dans la lutte contre le changement climatique, très suivi). Ce tweet est en effet accompagné d'une capture d'écran du site d'Europe 1, sur laquelle on voit effectivement un gros titre « $\mathrm{Du}$ tritium dans l'eau potable de millions de personnes selon une association " puis, en petits caractères, une relativisation des niveaux de contamination.

De nombreux messages sont dirigés contre le Parisien, dont les articles, d'abord alarmistes puis dénonçant la "fake news", avaient été largement partagés sur les médias sociaux: "Coucou @le_Parisien. C'est dommage d'oublier de dire que ces rumeurs viennent en grande partie de VOTRE article sur le sujet » (compte très suivi, ingénieur, membre de @voixdunucleaire; Figure 9), ou encore: "Ce n'est pas le Parisien qui a diffusé des informations alarmistes sur le tritium pour ensuite sortir un article où il déplorait des rumeurs alarmistes sur les réseaux sociaux?» (compte d'un chercheur en informatique, très suivi). Les médias sont accusés d'avoir choisi des titres alarmistes, visant à attirer le lectorat, au mépris de toute déontologie : "Une bêtise et un démenti ça fait deux articles qui tournent coco. \#LoiDeLaPresse \#Tritium » (compte d'un avocat, assez suivi). Pour ces utilisateurs, c'est ce traitement sensationnaliste qui aurait contribué à l'émergence et à la diffusion des messages relatifs à la potabilité de l'eau.

Figure 9. Tweet interpellant le Parisien à propos de sa responsabilité dans la diffusion de la rumeur.

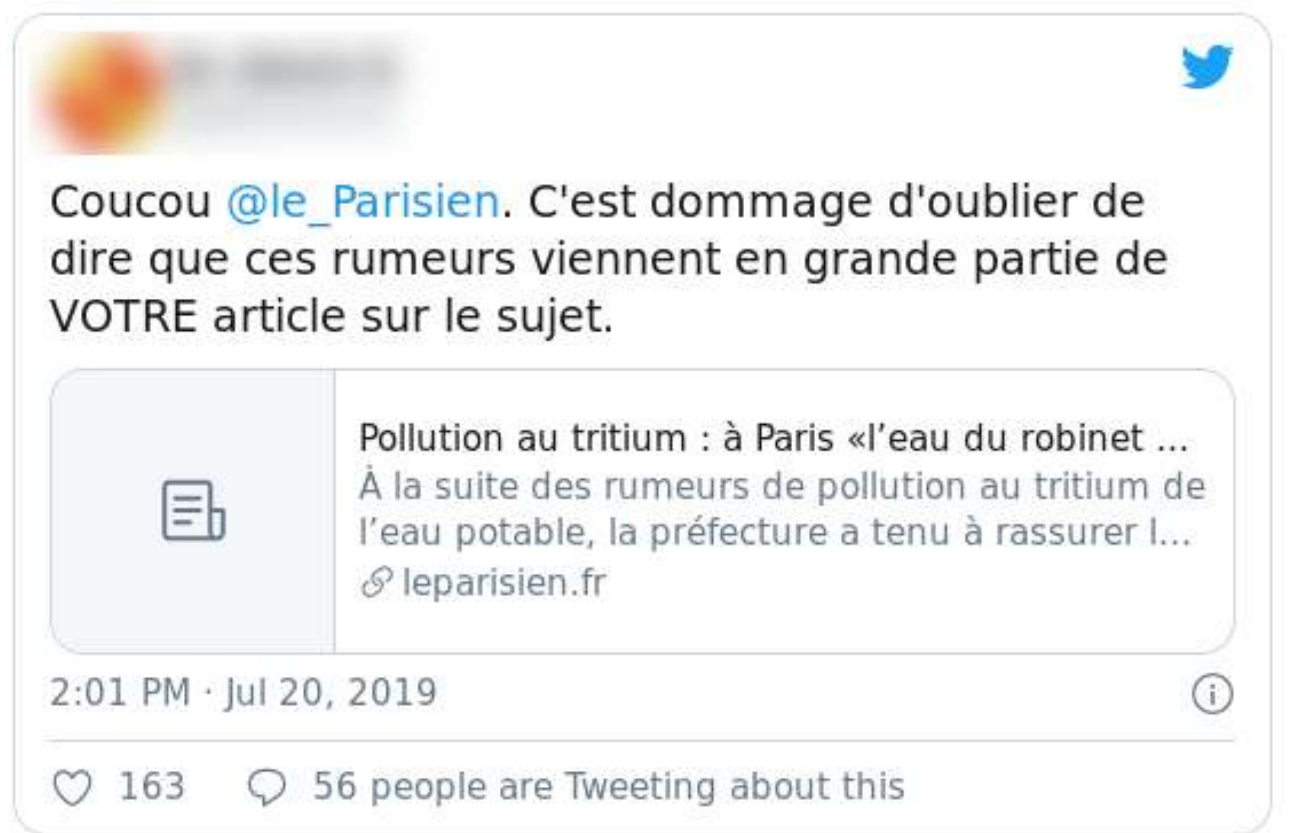

Les acteurs qui prennent l'initiative de dénoncer le partage d'informations erronées, ou toute autre action présumée déviante, ne le font pas sans raison: «pour crier au 
voleur, il faut y trouver un avantage ; c'est l'intérêt personnel qui pousse à prendre cette initiative » (Becker, 1985 : 146). La lutte contre les informations erronées présente un intérêt certain pour les médias traditionnels qui, souffrant d'un contexte économique défavorable et d'un important déficit de confiance auprès d'une bonne part de la population, y voient une opportunité de retrouver une place centrale sur le marché de l'information (Doutreix \& Barbe, 2019). Néanmoins, ce nouveau rôle pourrait les amener à exagérer l'importance du phénomène de malinformation, tout comme les policiers peuvent surestimer la prévalence de certains délits car « cette affirmation leur fournit une bonne raison pour maintenir l'existence des emplois qu'ils occupent " (Becker, 1985 : 181). De plus, la position des journalistes, à la fois juge et partie, a fait l'objet de critique, notamment à l'occasion de la création d'outils évaluant la fiabilité de sources, comme le Decodex du Monde (Badouard, 2017 : 47). Car pour les utilisateurs des médias sociaux, ces démarches sont vues comme des formes de « disqualification de ce qui paraît amateur et moins normé » (Doutreix \& Barbe, 2019). Dans cette controverse relative à l'origine de la rumeur, et face aux accusations des médias, l'intérêt des utilisateurs de Twitter n'est alors ni plus ni moins que la défense de leur légitimité à intervenir dans le débat public.

\section{Défiances et résistances}

39 Ce conflit relatif à l'attribution de la responsabilité de la rumeur s'inscrit dans une remise en cause, plus globale et plus ancienne, de certaines pratiques journalistiques. Ainsi, dans le corpus, l'affaire du tritium est reliée à d'autres controverses à propos desquelles le rôle des médias est critiqué. Le cas du glyphosate, dont la dangerosité avait fait l'objet d'une large couverture médiatique début 2019 alors qu'elle fait encore débat dans les milieux scientifiques, est fréquemment évoqué : "Ce que les journaux ont fait avec le Tritium, ils le font aussi avec le Glyphosate. Marchands de peurs » (compte sous pseudonyme, s'inscrivant dans la mouvance ultralibérale « $\mathrm{Ze}$ », très suivi). L'accumulation de comparaisons est utilisée pour dénoncer ce qui est vu, selon les utilisateurs, comme une stratégie commerciale ou de l'incompétence pure et simple : «@franceinfo Heu, c'est aussi sérieux que vos infos sur le tritium, le glyphosate ou sur l'astéroïde qui va nous tuer dans quelques mois ?!» (compte sous pseudonyme, suspendu à ce jour, rediffusant des messages hostiles à la gauche, assez suivi). La réfraction, processus par lequel un message partagé est recontextualisé ou relié à d'autres messages (Rieder, 2012), est utilisée pour généraliser la critique contre les médias, bien au-delà du seul cas du tritium. Ainsi, si la diffusion d'informations erronées peut constituer "l'expression d'une défiance virulente à l'égard des élites politiques et intellectuelles" (Badouard, $2017: 44$ ), on retrouve cette même défiance parmi ceux qui se mobilisent contre les rumeurs. Ce n'est pas la norme de vérité qui est ici critiquée, mais certaines des institutions qui, comme la presse, sont traditionnellement chargées de la faire respecter.

Cette défiance se nourrit des erreurs des médias, mais surtout du manque d'excuses ou d'autocritique lorsqu'elles sont découvertes : «Cette fake news. sur l'eau contaminée au tritium a fait du mal. Des gens ont peur de boire désormais. Les médias responsables de cette rumeur devraient s'excuser. » (compte d'un professionnel de la communication et blogueur, sujets politiques, très suivi). D'autant que, lorsqu'ils sont en position d'évaluateurs, comme dans le Decodex, les journalistes font preuve d'une sévérité variable : «Les fake news diffusées par certains médias traditionnels seront considérées 
comme des erreurs alors que les mêmes fake news, relayées par des sites alternatifs, seront portées à leur discrédit. » (Doutreix \& Barbe, 2019). L'application sélective des normes n'est pas une exception en matière de sanction de la déviance : « Le respect des normes est imposé sélectivement en fonction du type de personne, du moment et de la situation » (Becker, 1985 : 156). Et elle trouve une certaine symétrie dans les réactions des usagers des Twitter : là où les personnes ayant partagé les messages alarmistes sont peu inquiétées une fois qu'elles ont reconnu leurs torts ou supprimé leurs messages, les médias ne bénéficient pas de cette indulgence (Figure 10). Les références fréquentes à l'affaire du glyphosate, pourtant survenu plusieurs mois auparavant, montrent que les erreurs de la presse restent bien présentes dans les mémoires. En raison du rôle central que nos sociétés attribuent à la presse dans l'application de la norme de vérité, ses transgressions de cette même norme sont jugées comme intolérables, et sont particulièrement sanctionnées.

Figure 10. Message montrant comment la diffusion de titres alarmistes affecte durablement la crédibilité de la presse dans son rôle historique de garant des normes de vérité (compte rediffusant des contenus NoFakeScience et NoFakeMed, assez suivi).

\section{Donc la presse dans son ensemble qui a propagé la rumeur sur le \#tritium sans faire son boulot de critique et d'analyse veut nous expliquer aujourd'hui comment la rumeur s'est propagée... 열 2 is}

10:43 PM · Jul 21, 2019

\section{1 \& See other Tweets}

Le contexte est en outre favorable à la critique du manque de déontologie et des dérives sensationnalistes de la presse autour des thématiques scientifiques. En effet, quelques jours avant le communiqué de l'ACRO, un groupe de chercheurs, de journalistes et de vulgarisateurs, se réclament souvent du rationalisme ou de la zététique, avait publié une tribune intitulée "No Fake Science", demandant un traitement des sujets scientifiques qui permette « un débat public apaisé et rationnel ». Ce terme apparaît à de nombreuses reprises dans le corpus étudié, sous forme de hashtags dans les tweets : "L'intérêt de la création de \#NoFakeScience encore une fois démontrée » (compte d'un ingénieur, pharmacien, amateur de science, assez suivi), mais aussi dans les descriptions et pseudonymes de certains utilisateurs. La tribune a fait l'objet de critiques, à sa publication (Sénécat, 2019) et plus récemment (Foucart et al., 2020), en raison de sa bienveillance et de la proximité de certains signataires vis-à-vis des industries agroalimentaire ou nucléaire. Cette ambivalence transparaît dans le corpus, certains utilisateurs associés à l'industrie nucléaire se réclamant de NoFakeScience, tandis que d'autres en dénoncent le scientisme : «Les cadors de \#NoFakeScience vous expliqueront, comme pour le \#glyphosate : pas souci pour la santé humaine. Les seuils sont tellement stricts. Le consensus scientifique est tellement scientifique. Oubliant le droit de ne pas manger de tritium ou de cervelle de singe vivant.» (compte critiquant 
la politique du gouvernement LREM, assez suivi). Mais ces divergences portent principalement sur le rôle que la science devrait avoir dans la société, et ne remettent pas en question les critiques envers la presse. Ainsi, de la même manière que le terme «fake news » avait été utilisé pour afficher le caractère déviant des messages alarmistes, l'expression "no fake science " pourrait dans ce cas précis être considérée comme un label dénonçant les entorses à la norme que constitue la déontologie journalistique.

Les usagers de Twitter et la population dans son ensemble sont fréquemment vilipendés, par les partisans du rationalisme comme par d'autres, pour leur manque de compétence scientifique ou de recul critique :

«Le plus terrifiant dans cette histoire de \#tritium, au delà du phénomène maintenant classique d'une Fake-news qui se répand rapidement, c'est l'inculture scientifique absolue d'une vaste partie de la population. Je ne les blâme pas, c'est avant tout l'échec de l'école publique.» (compte d'un militant pour des causes sociales et écologistes, assez suivi, engagé dans la médiation scientifique, très suivi).

De nombreux messages font référence, de manière tantôt humoristique, tantôt sérieuse, à l'homéopathie et aux principes "pseudo-scientifiques" qui la sous-tendent : "Otez-moi d'un doute... Le tritium à dose homéopathique, ça doit protéger contre la radioactivité, non? Par contre, il faut penser à bien l'agiter.» (compte d'un professionnel de la santé engagé dans la politique, très suivi). Ces critiques parfois condescendantes ne sont toutefois pas sans lien avec la défiance qui s'exprime envers les médias : «Encore une histoire qui montre bien qu'il faut avoir un esprit critique et pas gober aveuglément ce que les médias balancent (sans connaitre réellement à fond le sujet bien souvent) \#tritium " (compte sous pseudonyme, développeur informatique, assez suivi). L'appel à l'esprit critique est ici jugé nécessaire parce que les gatekeepers traditionnels ne sont plus considérés comme fiables. Sans eux, il revient à chaque citoyen d'évaluer que les informations reçues sont conformes à la norme et de dénoncer celles qui ne le sont pas. Certains utilisateurs s'engagent effectivement dans des opérations d'analyse critique et en font bénéficier leurs abonnés : « 58 articles de presse analysés, 619 commentaires répertoriés L'article sur l'eau radioactive avance bien. Rendez-vous dans quelques jours/semaines pour un débriefing le plus exhaustif possible sur cette affaire » (compte sous pseudonyme, blogueur, engagé dans l'analyse critique des médias, très suivi). La popularité de ces citoyens devenus influenceurs, parfois difficiles à distinguer des médias alternatifs émergents, semblent indiquer que le moment est bien choisi pour « devenir média » à la place des médias.

\section{Conclusion}

Cet article visait à analyser le processus par lequel des discours jugés déviants sont dénoncés comme tels et combattus sur les médias sociaux. À travers l'étude des tweets relatifs au tritium dans l'eau, différents mécanismes ont pu être observés. L'articulation des niveaux de communication micro et macro (Bruns \& Moe, 2014) a permis une diffusion massive des démentis et de la pression sociale les accompagnant : les hashtags assurent une large visibilité aux messages et aux qualificatifs qui leur sont attribués (rumeurs, fake news, etc.), tandis que les mentions et réponses sont utilisées pour attirer l'attention d'utilisateurs ciblés ou les confronter directement. On constate que cette pression sociale induit des comportements de préservation et de ménagement de la face, notamment à l'égard des utilisateurs cédant à la pression sociale. Les pratiques de réfraction, parfois combinées à l'exhumation de messages plus anciens, sont quant à 
elle utilisées pour critiquer ceux, comme les médias, dont la déviance est jugée inexcusable. Ainsi, si les messages qualifiés de rumeurs ont pu connaître une diffusion rapide grâce aux médias sociaux, leur dénonciation s'est ensuite suivie d'une répression suffisamment large et féroce pour les juguler et marginaliser les contrevenants.

L'étude de la rumeur met également en lumière les multiples enjeux de pouvoir qui traversent le processus d'imposition des normes de vérité. Les controverses socioscientifiques portant sur le risque nucléaire ont, sans surprise, connu un nouveau rebond. Les factions anti- et pro-nucléaire, présentes et bien organisées sur les médias sociaux, ont dans un premier temps pu faire la démonstration habituelle de leur opposition. Celle-ci a néanmoins été vite éclipsée par les discussions relatives au traitement médiatique de la controverse. En effet, certains acteurs historiques, dont les médias, occupent une place centrale dans le processus de lutte contre la rumeur. Mais, dans le même temps, ces acteurs voient leur rôle remis en cause, en raison de leur positionnement pas toujours très clair et de leurs intérêts parfois contradictoires en matière de lutte contre les informations erronées. Cette dénonciation, associant pro- et anti-nucléaires, a également réuni des citoyens, des scientifiques, des journalistes aux motivations variées. Ainsi, la rumeur étudiée dans cet article a alimenté ou réactivé des controverses pré-existantes, occasionnant des jeux d'alliance complexes entre différents groupes d'acteurs : "Le problème de l'imposition du respect des normes se complique quand la situation voit s'affronter plus de deux groupes. Les arrangements et les compromis sont plus difficiles parce qu'il y a plus d'intérêts à satisfaire et les conflits risquent davantage d'être ouverts et sans solution » (Becker, 1985 : 151). De fait, la gestion des déviances informationnelles ne peut être étudiée indépendamment des tensions qui traversent la société au même moment, tant elles se nourrissent mutuellement.

La forte défiance qui s'exprime aujourd'hui envers les médias, dans le corpus étudié mais plus largement aussi, témoigne des recompositions en cours parmi les acteurs chargés de l'imposition des normes de vérité. Si la critique de la presse, et notamment des «partis pris spectaculaires» induits par la recherche d'audiences, n'est pas nouvelle (Lemieux, 2000), le paysage médiatique actuel est particulièrement mouvant. Les appels à l'esprit critique, de même que le rôle important assumé par certains utilisateurs dans la lutte contre la rumeur, sont autant de marques de l'affaiblissement des gatekeepers traditionnels. On peut néanmoins s'interroger sur la capacité des citoyens à assurer chacun, par eux-mêmes, l'application des normes de vérité. Le cas étudié montre plutôt l'apparition de nouvelles figures d'autorité, plus locales, construisant leur notoriété sur une certaine légitimité scientifique ou sur un travail de vulgarisation et de décryptage des controverses.

Cependant, ces figures médiatiques émergentes pourraient se trouver rapidement en compétition avec les plateformes de médias sociaux sur lesquelles elles s'expriment. En effet, leur rôle dans la détection et la suppression des contenus considérés comme déviants, tout comme les tergiversations relatives à l'interdiction des publicités à caractère politique ou des messages remettant en cause les résultats d'élections, semblent montrer une implication croissante des plateformes dans l'application des normes. Mais les entreprises qui possèdent ces plateformes n'ont ni les mêmes intérêts ni les mêmes obligations que la presse, émergente ou établie. Ce sont là deux directions très différentes que pourrait emprunter la gestion des déviances informationnelles dans les prochaines années: un renouvellement des formes et des déontologies 
journalistiques réactivant leur rôle de contre-pouvoir, ou un contrôle discrétionnaire délégué aux quelques entreprises se partageant le monopole des dispositifs de communication. Avec des implications importantes sur l'évolution des mécanismes d'imposition des normes de vérité, voire l'évolution de ces normes elles-mêmes.

\section{BIBLIOGRAPHIE}

ALGOTRANSPARENCY (s. d.). «AlgoTransparency.org », consulté le 09.04.2021. URL: https:// www.algotransparency.org/

ALLOING Camille \& VANDERBIEST Nicolas (2018). « La fabrique des rumeurs numériques. Comment la fausse information circule sur Twitter? », Le Temps des médias, 1, pp. 105-123.

BADOUARD Romain (2017). Le désenchantement de l'internet. Désinformation, rumeur et propagande, Limoges, FYP éditions.

BADOUARD Romain \& MABI Clément (2015). « Le débat public à l'épreuve des controverses », Hermes, La Revue, 71 (1), pp. 145-151.

BECKER Howard S. (1985). Outsiders. Études de sociologie de la déviance, Paris, Métailié.

BLUMER Herbert (1962). « Society as symbolic interaction », in Herman Nancy \& Reynolds Larry T. (eds.) Symbolic interaction: an introduction to social psychology, New York, General Hall, pp. 264-266.

BOUDIA Soraya (2007). « Naissance, extinction et rebonds d'une controverse scientifique », Mil neuf cent. Revue d'histoire intellectuelle, 25 (1), pp. 157-170.

BOYD danah (2016). C'est compliqué : les vies numériques des adolescents. Caen, C\&F éditions.

BRESLIN John G., PASSANT Alexandre \& DECKER Stefan (2009). The social semantic Web. New York, Springer.

BRUNS Axel \& KELLER Tobias (2020). « News diffusion on Twitter: comparing the dissemination careers for mainstream and marginal news ", in Social Media \& Society 2020 Conference.

BRUNS Axel \& MOE Hallvard (2014). «Structural layers of communication on Twitter », in Weller Katrin, Bruns Axel, Burgess Jean, Mahrt Merja \& Puschmann Cornelius (eds) Twitter and society, New York, Peter Lang, pp. 15-28.

BRUNS Axel \& STIEGLITZ Stefan (2012). « Quantitative approaches to comparing communication patterns on Twitter ", Journal of Technology in Human Services, 30 (3-4), pp. 160-185.

CARDON Dominique \& LEVREL Julien (2009). « La vigilance participative. Une interprétation de la gouvernance de Wikipédia », Réseaux, 154, pp. 51-89.

DAUPHIN Florian (2019). «Les fake news au prisme des théories sur les rumeurs et la propagande », Études de communication, 53 (2), pp. p. 15-32.

DI SCALA Emmanuella (2019). Science en société. Les représentations dans les controverses et les questions socio-scientifiques, Paris, L'Harmattan. 
DOUTREIX Marie-Noëlle \& BARBE Lionel (2019). «Légitimer et disqualifier : les fake news saisies comme opportunité de normalisation du champ journalistique », Études de communication, 53 (2), pp. 49-66.

FOUCART Stéphane, HOREL Stéphane \& LAURENS Sylvain (2020). Les gardiens de la raison. Enquête sur la désinformation scientifique, Paris, La Découverte.

FRAU-MEIGS Divina (2019). Faut-il avoir peur des fake news ?, Paris, La documentation française.

GOFFMAN Erving (1974). Les rites d'interaction, Paris, Minuit.

HAHN Kyu S., RYU Seungjin \& PARK Sungjin (2015). « Fragmentation in the Twitter following of news outlets: the representation of South Korean users' ideological and generational cleavage ", Journalism \& Mass Communication Quarterly, 92 (1), pp. 56-76.

HEIMBACH Irina, SCHILLER Benjamin, STRUFE Thorsten \& HINZ Oliver (2015). « Content virality on online social networks: Empirical evidence from Twitter, Facebook, and Google+ on German news websites ", in Proceedings of the 26th ACM Conference on Hypertext \& Social Media. ACM, pp. 39-47.

KAPFERER Jean-Noël (1987). Rumeurs, le plus vieux média du monde, Paris, Seuil.

KAPLAN Andreas M. \& HAENLEIN Michael (2010). « Users of the world, unite! The challenges and opportunities of Social Media », Business horizons, 53 (1), pp. 59-68.

LEMIEUX Cyril (2000). Mauvaise presse : une sociologie compréhensive du travail journalistique et de ses critiques, Paris, Métailié.

LEMIEUX Cyril (2007). «À quoi sert l'analyse des controverses ? ", Mil neuf cent. Revue d'histoire intellectuelle, 1, pp. 191-212.

MARWICK Alice E. (2018). «Why do people share fake news? A sociotechnical model of media effects », Georgetown Law Technology Review, 2 (2), pp. 474-512.

MCLUHAN Marshall (1968). Pour comprendre les médias: les prolongements technologiques de l'homme, Paris, Seuil.

MENDOZA Marcelo, POBLETE Barbara \& CASTILLO Carlos (2010). « Twitter Under Crisis: Can we trust what we RT? ", in Proceedings of the first workshop on social media analytics. ACM, pp. 71-79.

OTT Brian L. (2017). « The age of Twitter: Donald J. Trump and the politics of debasement ", Critical studies in media communication, 34 (1), pp. 59-68.

OXFORD DICTIONARIES (2016). « Word of the Year 2016 is... », consulté le 09.04.2021. URL: https:// en.oxforddictionaries.com/word-of-the-year.

PERKO Tanja, TURCANU Catrinel \& GEENEN Dries (2012). « Media reporting and changes in public opinion after Fukushima nuclear accident: Belgium as case study », International Journal of Nuclear Governance, Economy and Ecology, 3 (4), pp. 291-307.

RAYNES-GOLDIE Kate (2010). « Aliases, creeping, and wall cleaning: Understanding privacy in the age of Facebook », First Monday, 15 (1), consulté le 09.04.2021. URL: https://firstmonday.org/ojs/ index.php/fm/article/view/2775

RIEDER Bernhard (2012). " The refraction chamber: Twitter as sphere and network », First Monday, 17 (11), consulté le 09.04.2021. URL: https://firstmonday.org/ojs/index.php/fm/article/view/ 4199/3359.

ROPEIK David (2008). « Risk communication, more than facts and feelings », IAEA Bulletin, 50 (1), pp. 58-60. 
SEGAULT Antonin (2018). « Crossplatform references on social media: Methodological challenges and research avenues ", in Proceedings of the 2nd International Conference on Web Studies. ACM, pp. 52-55.

SEGAUlT Antonin, TAJARIOL Federico \& ROXIN Ioan (2017). « Production et diffusion de documents numériques via Twitter : le cas d'une fausse vidéo ", in Le Document ? Actes du 20e colloque international sur le document numérique. Europia, pp. 285-296.

SÉNÉCAT Adrien (2019). « Les évidences relatives de la tribune de No Fake Science sur l'information scientifique ", Le Monde, consulté le 09.04.2021. URL: https://www.lemonde.fr/les-decodeurs/ article/2019/07/26/les-evidences-relatives-de-la-tribune-de-no-fake-science-sur-l-informationscientifique_5493749_4355770.html

SIMON Justine, TOULLEC Bénédicte, BADOUARD Romain, BIGEY Magalie, COMPAGNo Dario, MERCIER Arnaud, ... SEBBAH Brigitte (2017). «L'influence des discours d'accompagnement sur le partage social. Identifier et analyser les discours d'escorte sur Twitter ", in Wigham Ciara R. \& Ledegen Gudrun (eds) Corpus de communication médiée par les réseaux. Construction, structuration, analyse, Paris, L'Harmattan, pp. 52-70.

SLOVIC Paul (1996). « Perception of risk from radiation », Radiation Protection Dosimetry, 68 (3-4), pp. 165-180.

SUROWIECKI James (2004). La sagesse des foules (Elen Riot, Trans), Paris, JC Lattès.

SUTTON Jeannette, PALEN Leysia \& SHKLOVSKI Irina (2008). « Backchannels on the front lines: Emergent uses of social media in the 2007 southern California wildfires », in ISCRAM 2008, Proceedings of the 5th International Conference on Information Systems for Crisis Response and Management, pp. 624-632.

TOPÇu Sezin (2006). « Nucléaire : de l'engagement « savant » aux contre-expertises associatives », Natures Sciences Sociétés, 14 (3), pp. 249-256.

TWITTER (2020a). « More about restricted uses of the Twitter APIs », consulté le 09.04.2021. URL: https://developer.twitter.com/en/developer-terms/more-on-restricted-use-cases.

TWITTER (2020b). « Search tweets », consulté le 09.04.2021. URL: https://developer.twitter.com/ en/docs/twitter-api/tweets/search/introduction.

vosoughi Soroush, ROY Deb \& ARAL Sinan (2018). « The spread of true and false news online », Science, 359 (6380), pp. 1146-1151.

WARDLE Claire (2017). « Fake news. It's complicated », First Draft, consulté le 09.04.2021. URL: https://firstdraftnews.org/latest/fake-news-complicated/

WILLIAMS Matthew L., BURNAP Pete \& SLOAN Luke (2017). « Towards an ethical framework for publishing Twitter data in social research: taking into account users' views, online context and algorithmic estimation ", Sociology, 51 (6), pp. 1149-1168.

\section{NOTES}

1. Sur ce point, voir également Badouard, dans ce même numéro.

2. Du tritium dans l'eau potable de millions de personnes, selon une association, sur Europe 1 : https://www.europe1.fr/sante/du-tritium-dans-leau-potable-de-millions-depersonnes-selon-une-association-3910186 
3. Du tritium décelé dans l'eau potable de 6,4 millions de personnes, sur Le Parisien : https://www.leparisien.fr/societe/du-tritium-retrouve-dans-l-eau-potable-de-6-4millions-de-personnes-17-07-2019-8118849.php

4. Six millions et demi de Français boivent une eau contaminée au tritium, sur France Bleu : https://www.francebleu.fr/infos/climat-environnement/6-millions-et-demi-defrancais-boivent-une-eau-contaminee-au-tritium-1563370365

5. Dans la description des comptes à l'origine des tweets cités, nous indiquerons, lorsque c'est le cas, si le compte publie sous pseudonyme (absence d'un nom de famille vraisemblable), s'il indique une activité professionnelle, s'il revendique une appartenance politique ou associative, ou si ses publications présentent des caractéristiques particulières (sujet récurrent, tonalité spécifique, etc.). Nous donnerons également une estimation de leur visibilité en utilisant les catégories suivantes : « peu suivi » pour ceux ayant moins de 100 abonnés, « assez suivi » entre 100 et 500 abonnés, et « très suivi » au-delà de 500 abonnés.

6. Banana Equivalent Dose, sur l'encyclopédie Wikipedia : https://en.wikipedia.org/ wiki/Banana_equivalent_dose

\section{RÉSUMÉS}

Dans l'imaginaire collectif, les médias sociaux semblent être un terrain favorable à la diffusion d'informations erronées. Cet article s'intéresse aux formes que prennent, sur ces plateformes, les processus de dénonciation des contenus considérés comme faux et donc déviants. Un corpus de tweets est étudié afin d'examiner la construction et la répression d'une rumeur portant sur la contamination de l'eau potable par du tritium, en juillet 2019. Les analyses montrent que les échanges se déroulant sur Twitter ont bien conduit à une imposition ferme des normes de vérité. Elles font en revanche apparaître de multiples enjeux de pouvoir, interrogeant notamment la légitimité des acteurs historiquement chargés de l'imposition de ces normes.

In our collective imagination, we tend to see social media as fertile grounds for the emergence and the spread of false information. This paper investigates, on these platforms, the process by which we condemn the contents that we consider false and therefore deviant. We built a corpus of tweets to study the making and the suppression of a rumor related to the contamination of tap-water with tritium, in July 2019 in France. Analysis shows that the discussions on Twitter actually led to an effective enforcement of the norms of truth. It however reveals multiple power struggles, especially regarding the legitimacy of the actors historically responsible for truth enforcement.

INDEX

Mots-clés : médias sociaux, fausses informations, rumeurs, déviance, normes de vérité Keywords : social media, fake news, rumors, deviance, norms of truth 
AUTEUR

ANTONIN SEGAULT

Université Paris Nanterre, Laboratoire Dicen-IdF 